cardiovascular events: a systematic review and meta-analysis. Circ Cardiovasc Qual Outcomes 2012: 5(5): 720-728.

3. National Institute for Health and Care Excellence. Continuous positive airway pressure for the treatment of obstructive sleep apnoea/hypopnoea syndrome. TA139. London: NICE, 2008. https://www. nice.org.uk/Guidance/TA139 (accessed 7 Mar 2016).

\section{Lack of a RCGP Research Committee}

College Officers commenting on my article on academic general practice ${ }^{1}$ refer to the Clinical Innovation and Research Centre (CIRC) and I agree it has achieved much in education and development. However, CIRC is not a research centre and is not seen nationally as such.

It is ironic that they cite the RCGP Research Paper of the Year as evidence of the College managing without a Research Committee. Corporate memory is not what it was! I invented this idea as Chairman of the College's Research Committee in the mid-1990s. The Research Committee/ Network and staff developed it vigorously as a new way of fostering research excellence, raising the RCGP's profile, and enabling the College to host an annual dinner for the research community at no cost to the College. So this award is actually evidence of what the College's former Research Committee could achieve.

The College's Research Committee is sorely missed. There is now no RCGP support for research-active general practices, although NHS policy is to promote research in all NHS settings, including NHS general practices. There is no RCGP campaign for opportunities for GPs and colleagues to do higher university degrees, which have reduced in recent years. The College's Research Committee successfully nominated a member of the primary care panel in the University Research Assessment Exercise, but that could not happen now.

As the College is richer than ever before, can the Research Committee be reinstated?

Denis Pereira Gray,

Chairman, RCGP Research Committee/ Network, 1993-1996.

E-mail: denis.pereiragraylabtinternet.com

\section{REFERENCE}

1. Pereira Gray D. Academic general practice: a viewpoint on achievements and challenges. $\mathrm{Br} J \mathrm{Gen}$ Pract 2015: D0I: 10.3399/bjgp15X687481. http://bjgp. org/content/65/640/e786

DOI: 10.3399/bjgp16X684505

\section{Discontinuation of benzodiazepines}

Following the recent clinical research article on the discontinuation of benzodiazepines, would like to encourage my colleagues. I have been working in a deprived area of Derby with relatively high levels of illegal drug use. Two and a half years ago the practice was prescribing benzodiazepines and Z-drugs at a level of $4 \%$ of the practice population (6500 patients). We discovered that some of these medications were entering the street market.

We initiated a firm protocol, called patients in for reductions, and discussed with our psychiatry colleagues the more difficult cases. With regular audit and review we have managed to reduced the regular burden of these drugs to $0.7 \%$ of our practice population and can expect to reduce that further to $0.15 \%$. As you can imagine this has taken a lot of effort and involved some difficult consultations and threatening behaviour, but the burden has been reduced not only for our patients but also for the wider community with no additional funding.

Naomi Walmsley,

GP, Derby.

E-mail: naomidaleladoctors.org.uk

\section{REFERENCE}

1. Vicens C, Sempere E, Bejarano F, et al. Efficacy of two interventions on the discontinuation of benzodiazepines in long-term users: 36-month follow-up of a cluster randomised trial in primary care. Br J Gen Pract 2016; DOI: 10.3399/ bjgp16X683485. http://bjgp.org/content/66/643/e85.

DOI: 10.3399/bjgp16X684517 